\title{
ESTUDIO POBLACIONAL DE MACROCYSTIS PYRIFERA (L.) C. AGARDH (LAMINARIALES: PHAEOPHYTA) EN AMBIENTES PROTEGIDO Y EXPUESTO AL OLEAJE EN TIERRA DEL FUEGO
}

\author{
POPULATION STUDY OF MACROCYSTIS PYRIFERA (L.) C. AGARDH \\ (LAMINARIALES: PHAEOPHYTA) IN WAVE PROTECTED AND \\ EXPOSED ENVIRONMENTS IN TIERRA DEL FUEGO
}

\author{
Jordi Plana ${ }^{1}$, Andrés Mansilla ${ }^{2}$, Mauricio Palacios² \& Nelso P. Navarro² \\ ${ }^{1}$ Programa de Magíster en Ciencias, Mención Manejo y Conservación de Recursos Naturales en Ambientes \\ Subantárticos, Facultad de Ciencias, Universidad de Magallanes. Email: jplana@aoniken.fc.umag.cl \\ ${ }^{2}$ Departamento de Ciencias y Recursos Naturales, Facultad de Ciencias, Universidad de Magallanes, \\ Casilla 113-D, Punta Arenas.
}

\begin{abstract}
RESUMEN
Se realizó un estudio poblacional sinóptico en poblaciones de Macrocystis pyrifera considerando distintos regímenes de exposición al oleaje en el Estrecho de Magallanes. Para un mejor manejo y análisis de la información obtenida, se utilizó el análisis SIG (Sistema de Información Geográfica). Los estudios se llevaron a cabo en Bahía Porvenir (53 $\left.18^{\prime} \mathrm{S} ; 70^{\circ} 24^{\prime} \mathrm{W}\right)$, Tierra del Fuego. Los muestreos se realizaron en transectas perpendiculares a la línea de costa, utilizándose tres cuadrantes de $1 \mathrm{~m}^{2}$ de área. Se estudiaron dos poblaciones, una localizada en una zona expuesta al oleaje y la otra en una zona protegida. Entre ambas poblaciones se compararon los siguientes parámetros biológicos: biomasa húmeda $\left(\mathrm{kg} \mathrm{m}^{-2}\right)$, densidad de estipes $\mathrm{m}^{2}$, porcentaje de individuos juveniles y adultos, y longitud de los individuos $(\mathrm{m})$. La población situada al interior de la bahía presentó mayores valores de biomasa ( $<<0,037)$, pero de igual forma una gran variabilidad entre individuos, marcándose diferencia dentro de una misma población, situación que no se observó en la población expuesta ( $>$ > 0,995), donde existió una uniformidad de relación a la biomasa por $\mathrm{m}^{2}$. La utilización de SIG en estudios de esta naturaleza facilita la visualización, monitoreo y análisis de la distribución de las poblaciones, en contraste con los métodos utilizados convencionalmente en estudios de campo.
\end{abstract}

Palabras Claves: Macrocystis pyrifera, Phaeophyta, estudio poblacional, Estrecho de Magallanes, Chile.

\section{ABSTRACT}

This study was carried out on populations of Macrocystis pyrifera in the Strait of Magellan found under different regimes of wave exposure. To better manage and analyze the data, GIS analysis was used. The study was carried out in Porvenir Bay $\left(53^{\circ} 18^{\prime} \mathrm{S} ; 70^{\circ} 24^{\prime} \mathrm{W}\right)$, Tierra del Fuego Island. The samples were collected in transects perpendicular to the shore, using three quadrats of $1 \mathrm{~m}^{2}$. Two populations were studied, one located in the area exposed to wave action and the other in a protected area. Between both populations the following biological parameters were compared: wet weight $\left(\mathrm{kg} \mathrm{m}^{-2}\right)$, density of stipes $\left(\mathrm{m}^{-2}\right)$, percentage of juvenile and adult individuals, and the length of individuals $(\mathrm{m})$. The population situated at the interior of the bay showed higher values of biomass $(\mathrm{p}<0.037)$, but at the same time a large variability between individuals, large differences within the same populations was not observed in the exposed population ( $p>0.995$ ), where there was a uniformity with regards to biomass per $\mathrm{m}^{2}$. Using GIS in the study of natural resources facilitates the visualization, monitoring and analysis of the populations, in contrast with methods conventionally used in field studies.

Keywords: Macrocystis pyrifera, Phaeophyta, population study, Strait of Magellan, Chile. 


\section{INTRODUCCION}

Macrocystis pyrifera (L.) C. Agardh (Lessoniaceae) es un alga parda, que presenta una distribución geográfica bipolar (North 1971), localizándose principalmente a lo largo de las costas del Pacífico de América del Norte (México, norte de Baja California y Alaska) y del Sur (Perú hasta Cabo de Hornos) (Alveal 1995), Sudáfrica, Australia, Nueva Zelanda (Druehl 2000). En Chile $M$. pyrifera se distribuye desde Tocopilla $\left(28^{\circ}\right.$ S) (Levring 1960) hasta el Cabo de Hornos $\left(55^{\circ}\right.$ S) (Ramírez \& Santelices 1991, Hoffmann \& Santelices 1997, Buschmann et al. 2004), sus poblaciones forman cordones continuos paralelos a la costa, de hasta $100 \mathrm{~m}$ de ancho, habitando preferentemente áreas protegidas y semiprotegidas del oleaje. La especie se caracteriza por formar densos bosques con ejemplares que alcanzan fácilmente los $30 \mathrm{~m}$ de longitud (Candia et al. 1979, Palacios \& Mansilla 2003) crecen fijas al sustrato rocoso mediante un grampón de hasta $40 \mathrm{~cm}$ de diámetro y $35 \mathrm{~cm}$. de altura.

M. pyrifera tiene una gran importancia ecológica en los lugares donde habita, siendo considerada como especie clave y paraguas para la coexistencia de otros organismos. Esta especie puede albergar y dar protección a una gran diversidad de organismos, incluyendo algas, peces, moluscos, entre otros (Adami \& Gordillo 1999; Ojeda \& Santelices 1984; Alveal et al. 1982; Palacios \& Mansilla 2003). En este aspecto cabe destacar que la presencia de $M$. pyrifera es crucial para mantener la organización y diversidad de comunidades ecológicas. Por otro lado, su elevado contenido en aminoácidos esenciales y de ácidos grasos, así como la calidad de sus proteínas y lípidos, lo hace comparable con las de otras fuentes vegetales (Cruz Suárez et al. 2000). Este recurso es utilizado como fuente de alginatos que a nivel mundial genera anualmente más de 250 millones de dólares (Vásquez 1999); actualmente se encuentra en estudio su utilización como suplemento alimenticio en la industria de salmonera (Mansilla et al. 2005a, 2005b), alimentación de abalones (Westermeier 2004) y erizos (Bustos et al. 1991). El creciente interés en la explotación de este recurso en la región de Magallanes hace necesario disponer de información que permita conocer la dinámi- ca y distribución de las poblaciones naturales así como los factores bióticos y abióticos que las regulan, permitiendo predecir cambios, tanto en su estructura como en su productividad a lo largo del tiempo, que permitan su utilización de manera sustentable, previendo que en un futuro no muy lejano estarán expuestas a una fuerte presión extractiva, como sucede actualmente con las algas carragenófitas (Norambuena 1996, Romo et al. 2006).

Como los Sistemas de Información Geográfica (SIG) basados en datos geográficos y sus atributos permiten combinar cartografía y bases de datos alfanuméricos asociados para estudios poblacionales de $M$. pyrifera, la utilización de estos sistemas facilita el análisis espacial de la información, aumentando la eficiencia de los métodos utilizados convencionalmente en trabajos de terreno (Bushing 1997) y permitiendo la integración de la información obtenida con la localización espacial y cartográfica del área de estudio.

\section{MATERIALES Y METODOS}

El estudio fue realizado en junio de 2002 (invierno) en Tierra del Fuego, específicamente en Bahía Porvenir $\left(53^{\circ} 18^{\prime} \mathrm{S} ; 70^{\circ} 24^{\prime} \mathrm{W}\right)$, distante aproximadamente $35 \mathrm{~km}$ de Punta Arenas (Fig. 1A). Bahía Porvenir es un lugar ideal para realizar este tipo de estudios, ya que presenta poblaciones de $M$. pyrifera a poca profundidad (< $5 \mathrm{~m}$ ), tanto dentro de la bahía como externamente a ella, posibilitando la comparación de poblaciones expuestas y protegidas al oleaje.

\section{Descripción del ÁREa de estudio}

La región se caracteriza por estar en el límite transaccional entre el clima trasandino y el clima de estepa frío, recibiendo influencias de ambos. Las temperaturas medias anuales se encuentran entorno de $\operatorname{los} 5,8^{\circ} \mathrm{C}$. La precipitación media anual en la Región de Magallanes fluctúa entre los 400 a $620 \mathrm{~mm}$, y los registros más altos de precipitación ocurren en verano y otoño (Pisano 1977). La temperatura superficial del agua decrece considerablemente durante la época invernal (junio/julio) registrándose valores que varían entre 3,8 y $5,2{ }^{\circ} \mathrm{C}$ (Iriarte et al. 2001). 

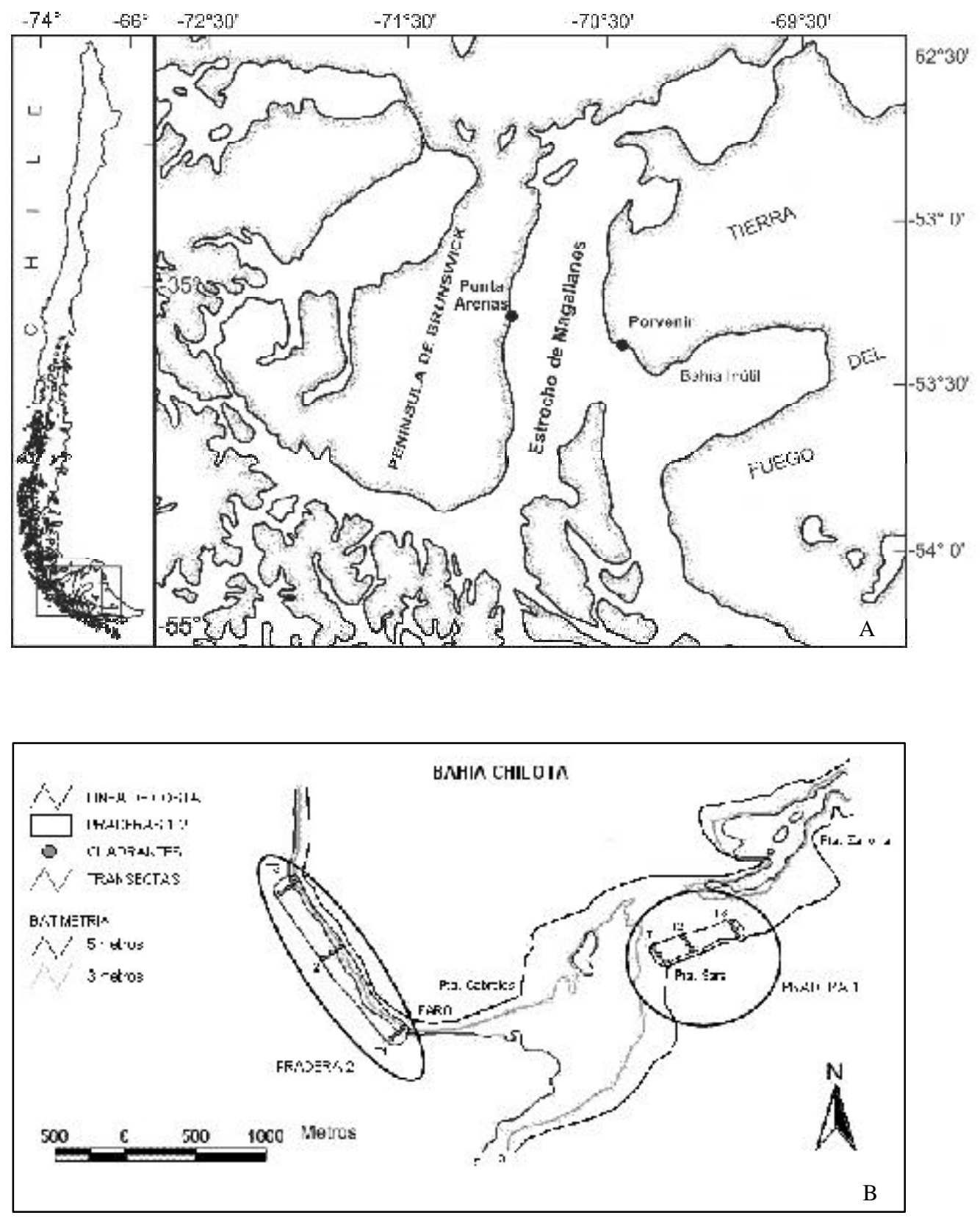

Figura 1. (A) Ubicación geográfica de las Bahías Porvenir, Tierra del Fuego. Layout (ArcView 3.2); (B) Representación de Bahía Porvenir, Tierra del Fuego $\left(53^{\circ} 18^{\prime} \mathrm{S} ; 70^{\circ} 24^{\prime} \mathrm{W}\right)$ con la localización de las poblaciones de M. pyrifera estudiadas, las transectas y los cuadrantes donde se realizaron los muestreos.

Figure 1. Geographic location of Porvenir Bays, Tierra del Fuego. Layout (ArcView 3,2). Representation of Porvenir Bay, Tierra del Fuego (53 18 ' S; $70^{\circ} 24^{\prime} \mathrm{W}$ ) with the location of the M. pyrifera populations transects and quadrants studied. 
Para el estudio fueron consideradas dos poblaciones de $M$. pyrifera, una situada en el interior de Bahía Porvenir (protegida al oleaje, denominada Población 1), y la otra situada en el exterior, cerca de la salida de la bahía (expuesta al oleaje, denominada Población 2). Previo a la realización de los muestreos se procedió a delimitar el área de las dos poblaciones mediante un Sistema de Posicionamiento Global (GPS). La delimitación de las poblaciones fue realizada mediante buceo autónomo. Para cada población se establecieron 3 transectas perpendiculares a la línea de costa, dentro de las cuales se establecieron 3 estaciones donde se realizaron muestreos utilizándose un cuadrante de $1 \mathrm{~m}^{2}$. Las variables cuantificadas en cada cuadrante fueron: biomasa húmeda $\left(\mathrm{kg} . \mathrm{m}^{-2}\right)$ y numero de estipes por $\mathrm{m}^{2}$, porcentaje de individuos juveniles o adultos (algas superiores a $2 \mathrm{~m}$ de longitud y con más de cuatro estipes) (Dayton et al. 1992) y longitud total de las algas, medida como la longitud máxima desde la parte inferior del grampón hasta el extremo de la fronda más alejada del mismo (m).

Para verificar diferencias entre las poblaciones estudiadas se aplicaron análisis de varianza (ANOVA), utilizando el software Statistica 5.0. En el caso de diferencias significativas, fue aplicado un test a posteriori de Newman-Keul (Zar 1999). Todas las conclusiones están basadas en un nivel de confianza de $95 \%(\mathrm{p}<0,05)$.

Los datos recopilados fueron introducidos en un entorno SIG, utilizando el software ArcView 3.2, del siguiente modo: primero se procedió a la digitalización de la carta náutica 1142 del Servicio Hidrográfico de la Armada de Chile, para luego ser geográficamente referenciada. Sobre este mapa se introdujeron los distintos "layers" correspondientes a cobertura de líneas para la línea de costa para la batimetría a 3 y 5 metros. Sobre el mapa obtenido, se procedió a introducir los "layers" correspondientes a las poblaciones y los muestreos, partiendo de la ubicación geográfica (latitud y longitud) de los mismos. Posteriormente se ingresaron la cobertura de puntos (cuadrantes), cobertura de líneas (transectas) y cobertura de polígonos (poblaciones). Finalmente, asociados a los anteriores se ingresaron las tablas de datos con los parámetros estudiados para cada cuadrante, para cada transecta y para cada una de las poblaciones. La utilización de esta herramienta nos permite una rápida localización y visualización de la zona de estudio y de las praderas de M. pyrifera, así como de las distintas transectas y cuadrantes de muestreo.

\section{RESULTADOS}

Biomasa húmeda $\left(\mathrm{kg} \mathrm{m}^{-2}\right)$ y densidad de estipes (estipes $\mathrm{m}^{-2}$ ).

La población protegida (población 1) presentó una biomasa húmeda promedio notoriamente mayor $\left(102,9 \pm 9,9 \mathrm{~kg} \mathrm{~m}^{2}\right)$ que en la población $2(83,5 \pm$ $5,1 \mathrm{~kg} \mathrm{~m}^{2}$ ) (Tabla I), estas diferencias fueron estadísticamente significativas (g.l=1; $\mathrm{F}=9,38 ; \mathrm{p}=$ 0,037) (Tabla III). Se observó también que en la población 1 los valores de biomasa por transecta tienen una mayor variabilidad con respecto a los de la población 2 (Tabla II). En la población 1 fueron observadas diferencias significativas entre los valores de biomasa obtenidos por transecta $(\mathrm{g} .1 .=2 ; \mathrm{F}=$ 8,21; $p=0,019$ ) (Fig. 2). Las principales diferencias fueron observadas entre las transectas T1 y T2 ( $\mathrm{p}=$ 0,017), T1 y T3 ( $p=0.023)$, con mayores valores de biomasa en $\mathrm{T} 3$ llegando a $96,3 \pm 7 \mathrm{~kg} \mathrm{~m}^{2}$, mientras que la menor biomasa se observó en T1 con 114,2 \pm $6,8 \mathrm{~kg} \mathrm{~m}^{-2}$ (Tabla II), sin embargo no se observaron diferencias entre las distintas estaciones de muestreo por transecta (g.l $=2 ; \mathrm{F}=0,119 ; \mathrm{p}=0,889)$. Dentro de la población 2, no se observaron diferencias significativas entre los valores de biomasa por transecta (g.l $=2 ; \mathrm{F}=0,004 ; \mathrm{p}=0,995)$, ni estación de muestreo (g.l= 2; $\mathrm{F}=0,18 ; \mathrm{p}=0,837$ ) (Fig. 2).

La densidad de las poblaciones (medida como estipes por $\mathrm{m}^{2}$ ) sigue un patrón similar al de la biomasa húmeda. De este modo se encuentra un mayor número de estipes por $\mathrm{m}^{2}$ en la población protegida (población 1) así como una mayor variabilidad del parámetro dentro de cada transecta (Tabla II). Los valores para la población expuesta son menores y más uniformes en las mismas (Tabla II). La densidad en la población 1 no presentó una distribución homogénea, siendo la transecta 2 la que presentó mayor densidad promedio de estipes $\left(760 \pm 256\right.$ estipes $\left.\mathrm{m}^{-2}\right)$ y la transecta 1 la de menor densidad (338 \pm 131 estipes $\mathrm{m}^{-2}$ ) (Tabla II, Fig. 3A). En la población 2 la transecta 1 (más cercana a la boca de la bahía), presentó los menores valores de densidad de estipes $\mathrm{m}^{-2}\left(162,6 \pm 36\right.$ estipes $\left.\mathrm{m}^{-2}\right)$. La mayor densidad de estipes se registró en la transecta 3 ( $249 \pm 26$ estipes $\mathrm{m}^{-2}$ ) (Tabla II, Fig. 3B). 
Gayana 71(1), 2007

TABla I. Promedios totales de biomasa húmeda $\left(\mathrm{kg} \mathrm{m}^{-2}\right)$, densidad de estipes (estipes $\left.\mathrm{m}^{-2}\right)$ y $\%$ de juveniles y adultos presentes en las poblaciones 1 y 2 de M. pyrifera en Bahía Porvenir, Región de Magallanes.

TABLE I. Total average of wet weight $\left(\mathrm{kg} \mathrm{m}^{-2}\right.$ ), density of stipes (stipes $\mathrm{m}^{-2}$ ) and $\%$ of youthful and adult plant presents in populations 1 and 2 of M. pyrifera in Porvenir Bay, Region of Magellan.

\begin{tabular}{lcc}
\hline Población & $\begin{array}{c}\text { Biomasa húmeda } \\
\left(\mathrm{kg} \mathrm{m}^{-2}\right)\end{array}$ & $\begin{array}{c}\text { Densidad de estipes } \\
\left(\text { estipes } \mathrm{m}^{-2}\right)\end{array}$ \\
\hline Protegida (1) & $102,9 \pm 9,9$ & $488 \pm 281$ \\
Expuesta (2) & $83,5 \pm 5,1$ & $209 \pm 45$ \\
\hline
\end{tabular}

TABLA II. Cuadro comparativo de los parámetros estudiados en dos poblaciones de M. pyrifera, al interior (población 1) y al exterior (población 2) por transecta de Bahía Porvenir, Región de Magallanes.

TABLE II. Comparative picture of some parameters of two natural populations of $M$. pyrifera, one of them located in inner Porvenir Bay (population 1) and the other located outside of the bay (population 2), Region of Magellan.

\begin{tabular}{cccccc}
\hline Población & Transecta & $\begin{array}{c}\text { Biomasa húmeda } \\
\left(\mathrm{kg} \mathrm{m}^{-2}\right)\end{array}$ & $\begin{array}{c}\text { Densidad de estipes } \\
\left(\text { estipes }^{-2}\right)\end{array}$ & $\begin{array}{c}\% \\
\text { juveniles }\end{array}$ & $\begin{array}{c}\% \\
\text { adultos }\end{array}$ \\
\hline Protegida & T1 & $114,2 \pm 6,8$ & $338 \pm 131$ & 5 & 95 \\
$(1)$ & T2 & $98,3 \pm 3,7$ & $760 \pm 256$ & 13 & 87 \\
& T3 & $96,3 \pm 7$ & $367 \pm 258$ & 45 & 55 \\
\hline Expuesta & T1 & $77,8 \pm 4,7$ & $162 \pm 36$ & 60 & 40 \\
$(2)$ & T2 & $86,6 \pm 1,9$ & $216 \pm 21$ & 44,4 & 55,6 \\
& T3 & $86 \pm 2,4$ & $249 \pm 26$ & 30 & 70 \\
\hline
\end{tabular}

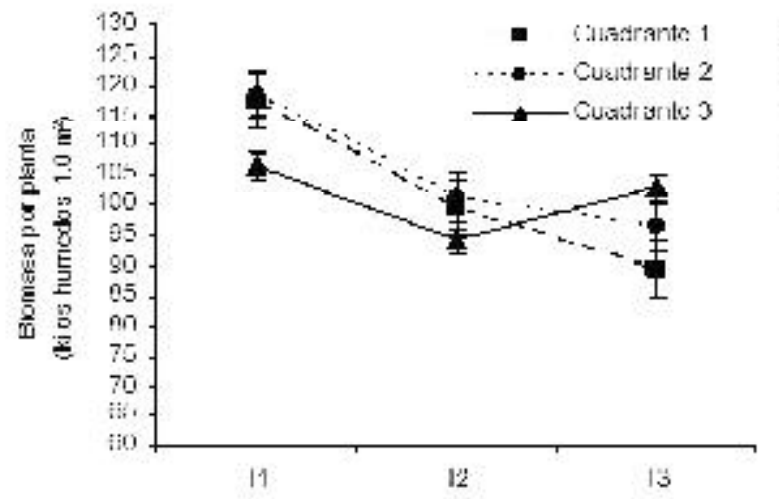

A Población 1 (protegida)

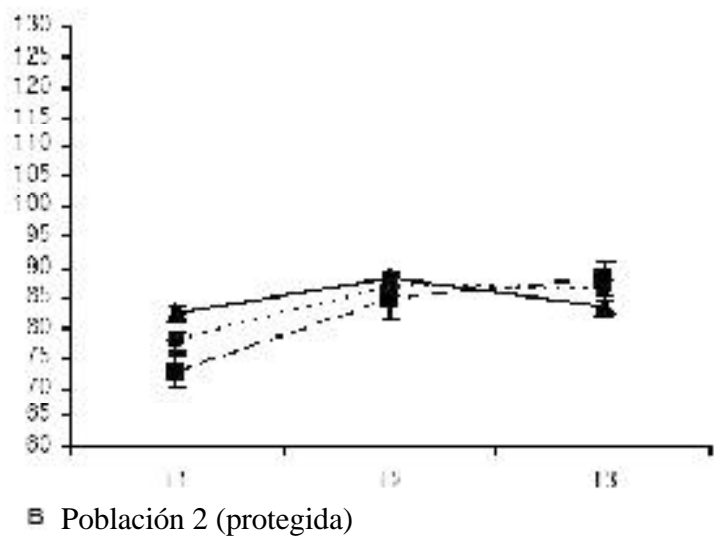

B Población 2 (protegida)

FIGURA 2. Distribución de biomasa húmeda $\left(\mathrm{kg} \mathrm{m}^{-2} \pm \mathrm{d}\right.$.S $)$ en cada transecta para la población protegida (A) y expuesta al oleaje (B) de M. pyrifera en Bahía Porvenir, Tierra del Fuego.

FIGURE 2. Distribution of wet weight $\left(\mathrm{kg} \mathrm{m}^{-2} \pm\right.$ s.d.) from each transect for the protected (A) and wave exposed (B) populations of M. pyrifera in Porvenir Bay, Tierra del Fuego Island. 


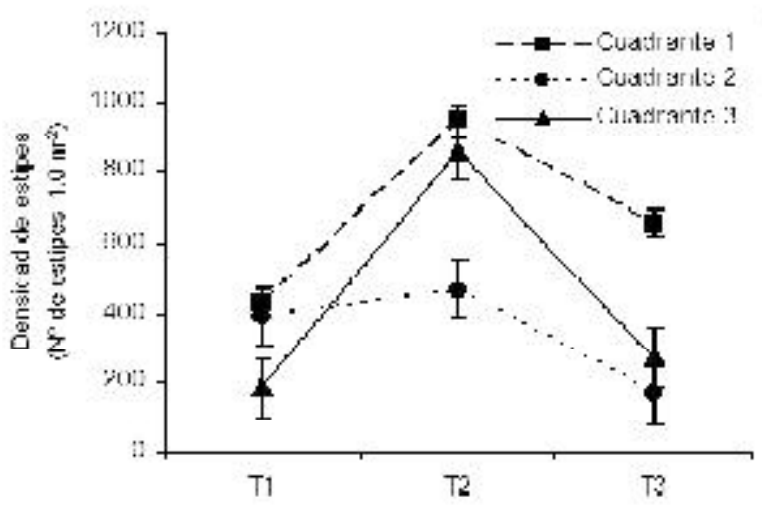

A. Población 1 (protegida)

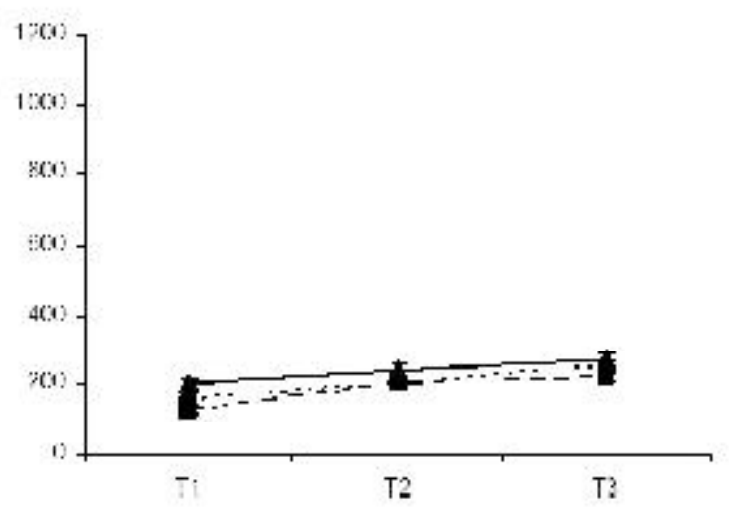

E Población 2 (protegida)

FIGURa 3. Distribución en la densidad de estipes $\left(\mathrm{N}^{\circ}\right.$ estipes $\mathrm{m}^{-2} \pm$ d.s) en cada transecta para la población protegida (A) y expuesta al oleaje (B) de M. pyrifera en Bahía Porvenir, Tierra del Fuego.

FIGURE 3. Distributions of the stipe density ( $\mathrm{N}^{\circ}$ estipes $\mathrm{m}^{-2} \pm$ s.d.) from each transect for the protected (A) and wave exposed (B) populations of $M$. pyrifera in Porvenir Bay, Tierra del Fuego Island.

No obstante lo anterior, el análisis de varianza no mostró diferencias significativas entre los valores de densidad de ambas poblaciones $(\mathrm{g} . \mathrm{l}=1 ; \mathrm{F}=4,06 ; \mathrm{p}=$ 0,113 ) (Tabla III), ni entre las transectas de cada una de las praderas (población 1 g.l=2; $\mathrm{F}=3,34 ; \mathrm{p}=0,105$ y población 2 g.l= 2; F= 0,032; p=0,967).

Porcentaje De JUVEnILES

El porcentaje de juveniles presenta tendencias distintas en ambas poblaciones. En la población protegida (población 1) se observó un mayor porcentaje de individuos juveniles, especialmente en la transecta situada en la zona más al interior de la bahía (T3), llegando a valores de $45 \%$ del total de la población (Tabla II, Fig. 4A), mientras que en la población expuesta (población 2) el mayor porcentaje de juveniles fue observado en la T1 con $60 \%$ (cercana a la boca de la bahía ), disminuyendo hacia el norte (T2= $44 \%$ y T3=30 \%), ubicadas en zonas más expuestas al oleaje y de mayor hidrodinamismo (Tabla II, Fig. 4B ). Sin embargo, dichas variaciones en los porcentaje de juveniles no son significativamente diferentes (g.l= 2; F= 0,050; p=0,833) (Tabla III), aun cuando existe una distribución especial para cada población en la proporción de individuos juveniles.

FRECUENCIA DE TALLA EN TRANSECTAS

En la población 1 se observa que la frecuencia de tallas presenta tendencia a una distribución normal centrada en tamaños entre 2 y 6 m (Fig. 5A). Esta tendencia no se observa en la población 2. Esta última posee individuos aglomerados en dos clases de tallas de 0-2 m y 6-10 m (Fig. 5B). En la población 1, los rangos de tallas adultas (sobre $2 \mathrm{~m}$ de longitud y más de cuatro estipes) se concentran en la transecta 2 , en tanto los individuos por sobre los $6 \mathrm{~m}$ de longitud se concentran en la transecta 1 (Fig. 5A). En la población 2, la distribución de tallas es relativamente homogénea con excepción de la transecta 3, donde se observaron individuos por sobre $\operatorname{los} 10 \mathrm{~m}$ de longitud (Fig. 5B).

No obstante lo anterior el análisis estadístico (ANOVA) de la información de terreno nos indicó la no existencia de diferencias significativas entre las poblaciones con relación a las tallas de los individuos (g.l=1; $\mathrm{F}=1,05 ; \mathrm{p}=0,305)$. Al realizar un análisis estadístico de población 1 se determinó la no existencia de diferencias significativas en relación a las longitudes de los individuos ( $\mathrm{p}>0,05$ ) (Fig. 6A). Para la población expuesta (población 2) se determinaron diferencias significativas entre los individuos muestreados ( $\mathrm{g} .1=2 ; \mathrm{F}=3,91 ; \mathrm{p}=0,023)$, dichas diferencias estadísticas fueron marcadas entre las transectas 1 y $3(\mathrm{p}=0,015)$, mientras que en las demás transectas no se determinaron dife rencias significativas (T1-T 2 p=0,188; T2-T3 p=0,137) (Fig. 6B). 


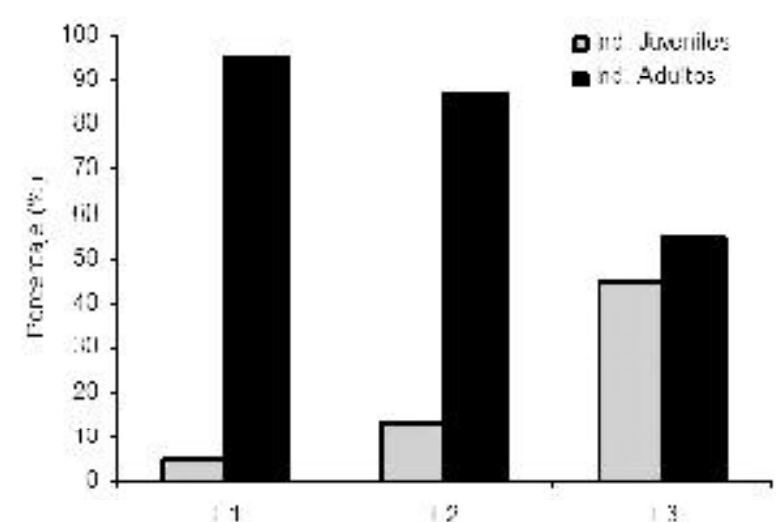

A Población 1 (protegida)

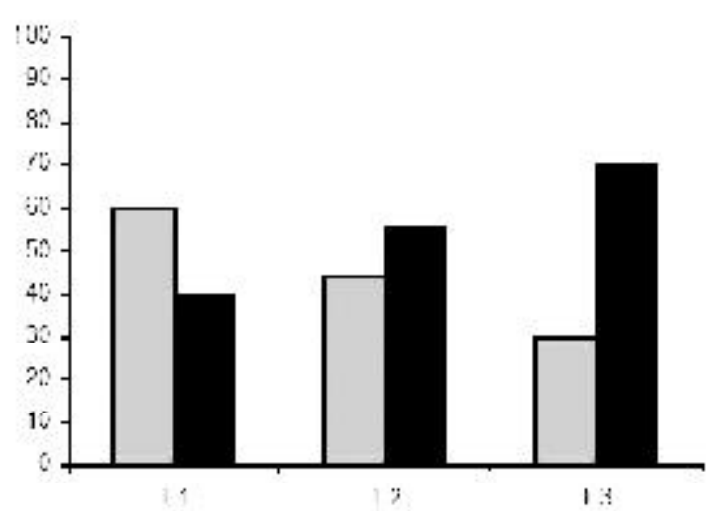

B Población 2 (protegida)

FIgURA 4. Porcentaje de juveniles y adultos de M. pyrifera en las transectas de dos poblaciones (A) protegida y (B) expuesta del oleaje en Bahía Porvenir, Tierra del Fuego.

Figure 4. Percentage of juvenile and adult M. pyrifera from each transects for the protected (A) and wave exposed (B) in Porvenir Bay, Tierra del Fuego Island.

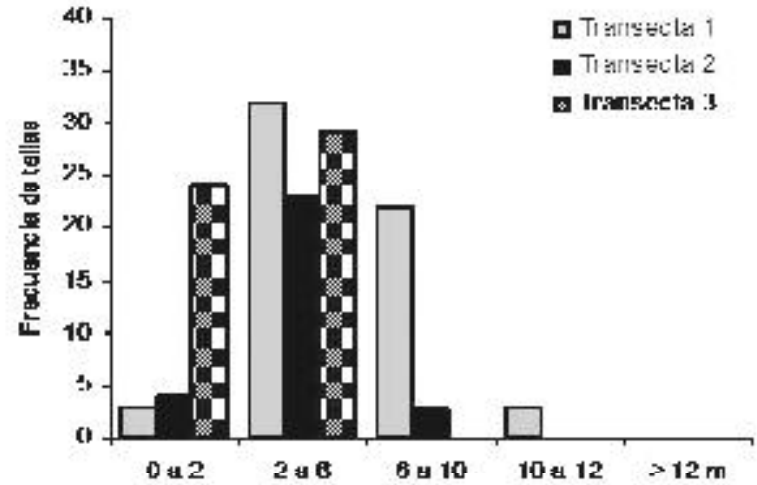

Población 1 (protegida)

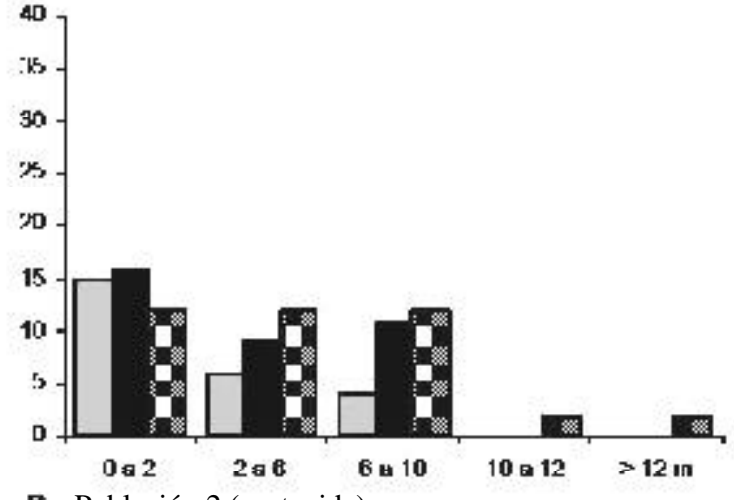

B Población 2 (protegida)

Figura 5. Frecuencia de tallas de M. pyrifera de dos poblaciones diferentes. (A) Población 1: protegida del oleaje; (B) Población 2: expuesta al oleaje. En Bahía Porvenir.

FIGURE 5. Frequency of statures of M. pyrifera for different populations. (A) Population 1: wave protected population; (B) Population 2: wave exposed population. In Porvenir Bay, Tierra del Fuego Island. 
Estudio poblacional de M. pyrifera en Tierra del Fuego: Plana, J. ET AL.

TABLA III. Análisis de varianza (ANOVA) para las interacciones de biomasa húmeda $\left(\mathrm{kg} \mathrm{m}^{-2}\right)$, densidad de estipes $\mathrm{m}^{-2}$, longitud promedio por individuos $(\mathrm{m})$ y porcentaje de juveniles presentes entre las poblaciones de $M$. pyrifera estudiadas $(\mathrm{p}<0,05)$.

TABLE III. Analysis of variance (ANOVA) for the interactions of wet weight $\left(\mathrm{kg} \mathrm{m}^{-2}\right)$, density of stipes $\mathrm{m}^{-2}$, average individual length $(\mathrm{m})$ and percentage of juveniles present in the populations of M. pyrifera that were studied $(\mathrm{p}<0.05)$.

\begin{tabular}{lccc}
\hline INTERACCIÓN & g.l & $\mathrm{F}$ & p-level \\
\hline Biomasa húmeda $\left(\mathrm{kg} \mathrm{m}^{-2}\right)$ & 1 & 9,382 & 0,0375 \\
Densidad de estipes $\left(\right.$ estipes $\left.\mathrm{m}^{-2}\right)$ & 1 & 4,069 & 0,1138 \\
Longitud de individuos (m) & 1 & 1,056 & 0,3050 \\
\% de juveniles & 2 & 0,050 & 0,8333 \\
\hline
\end{tabular}
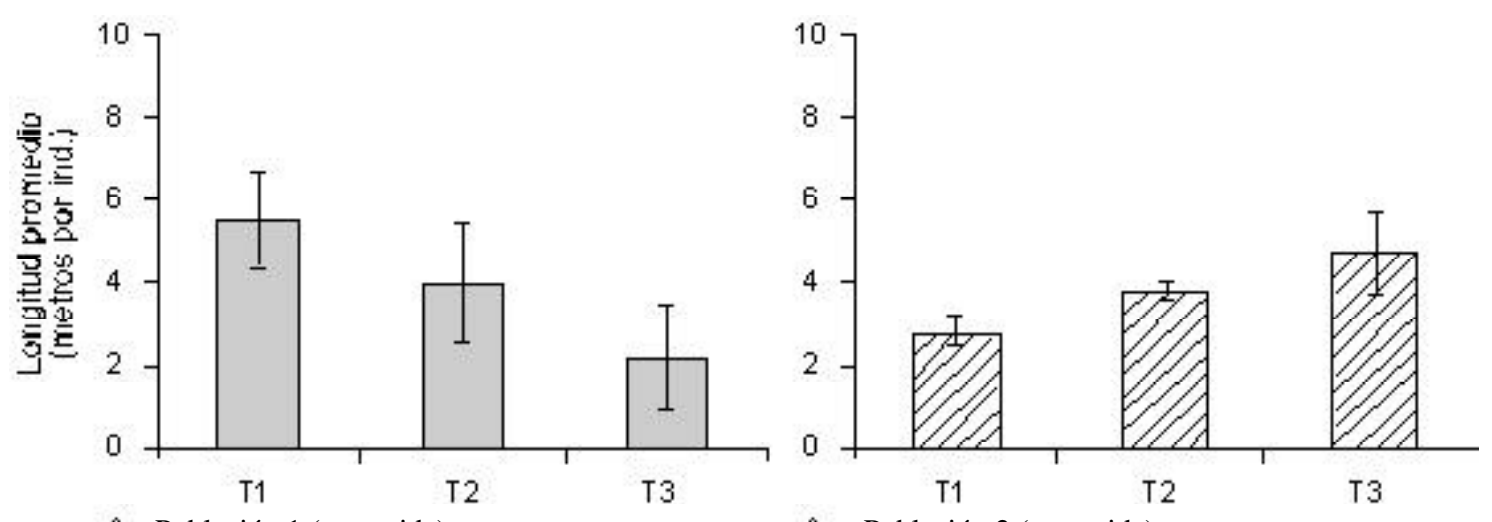

A. Población 1 (protegida)

A Población 2 (protegida)

FiguRa 6. Distribución de tallas promedio (metros \pm d.s) por transecta para la población protegida (A) y expuesta al oleaje (B) de M. pyrifera en Bahía Porvenir, Tierra del Fuego.

FIGURE 6. Distribution of average size (meters \pm s.d.) per transect for the protected (A) and wave exposed (B) populations of M. pyrifera in Porvenir Bay, Tierra del Fuego Island.

\section{DISCUSION Y CONCLUSIONES}

La información obtenida de los muestreos y vinculadas a los datos geográficos representados en el entorno SIG (ArcView 3.2) facilita la organización y el análisis espacial y temporal de la información obtenida en terreno.

El estudio poblacional sinóptico basado en la comparación de dos poblaciones de $M$. pyrifera de Bahía Porvenir permitió observar que, en la población situada al interior de la bahía (población 1), el número de individuos adultos aumenta, desde el interior hacia la boca de la bahía (de este a oeste). Se pudo observar además que el mayor porcentaje de ejemplares juveniles se encuentra en zonas con un menor hidrodinamismo (T3), donde tal vez las zoosporas, gametofitos y cigotos de $M$. pyrifera pueden asentarse mas fácilmente, siendo afectados en menor grado por las corrientes y el oleaje. Estos resultados concuerdan con la variación de la biomasa $\left(\mathrm{kg} \mathrm{m}^{-2}\right)$ entre las transectas, que disminuye a medida que nos desplazamos hacia el interior de la bahía.

Los resultados para la población situada en la zona exterior de Bahía Porvenir (población 2) muestran que existen diferencias en las tallas de los individuos muestreados entre las transectas $(\mathrm{p}=$ 0,023 ). En esta población, el mayor número de ejemplares juveniles se encuentra en la zona más cercana a la boca de la bahía, donde posiblemente 
la disponibilidad de zoosporas para asentamiento sería mayor debido a que esta zona recibe esporas que provendrían tanto de la población 2 como desde la población existente al interior de la bahía,. Por otro lado, se observó una tendencia al aumento en el tamaño de los individuos (> $12 \mathrm{~m}$ ), en la población situada en el sector expuesto de la bahía, donde probablemente existiría un incremento en la concentración de nutrientes, que favorecería el desarrollo vegetativo de las plantas como lo menciona Wheeler 1980, 1982; Gerard 1982. La densidad de estipes por $\mathrm{m}^{2}$ encontradas en las poblaciones de $M$. pyrifera de Magallanes presenta una tendencia similar a la densidad de plantas descritas por Buschmann et al. 2004, quienes registran mayor número de individuos por área ( $>15$ plantas 0,25 $\mathrm{m}^{2}$ ) en poblaciones protegidas en comparación con poblaciones expuestas que no sobrepasan de 4 plantas $0,25 \mathrm{~m}^{2}$. Sin embargo, es importante resaltar que parece más pertinente relacionar tanto el número de plantas o estipes por área con los valores de abundancia (biomasa), que permitirían comprender mejor la magnitud en términos de disponibilidad de recursos poblacionales.

En la población 1, situada al interior de la bahía, la presencia de un mayor porcentaje de individuos juveniles en las zonas más protegidas al oleaje (T3) concuerda con los resultados obtenidos por Graham et al. 1997 para estudios poblacionales de $M$. pyrifera en California Central (Península de Monterrey) donde los autores describen que en zonas protegidas las poblaciones juveniles son continuas y estables en el tiempo, ya que también lo es el dosel formado por las algas de mayor tamaño, si este disminuye su densidad, se estimula el rápido crecimiento de los juveniles que pasan en poco tiempo a estado adulto (3 meses), lo que se reafirma en el estudio realizado por Buschmannet al.2004, quienes determinaron que en poblaciones protegidas de M. pyrifera del sur de Chile el reclutamiento de juveniles ocurriría durante el invierno (julio), con lo cual aumenta la proporción de individuos juveniles dentro de una población. En cambio en las zonas expuestas al oleaje, el dosel formado por las algas adultas es inestable, lo que produce la existencia de condiciones irregulares de luz en el fondo, esto conlleva que el número de juveniles sea mas inestable e intermitente (Graham et al 1997). Estos datos concuerdan con la distribución de tallas de las algas dentro de la población 1 , pero no cuando compara- das las dos poblaciones entre ellas, ya que en la zona expuesta (población 2) los porcentajes de juveniles $\mathrm{y}$ adultos son semejantes, mientras que en la población 1 dominan las algas adultas, debido a que existiría menor penetración de luz, que permitiría el crecimiento de reclutas que se desarrollan bajo el dosel formado por las plantas adultas.

\section{AGRADECIMIENTOS}

Este estudio fue realizado con el apoyo y financiamiento del Proyecto Fondef DO1I1164 "Producción de Harina de Macroalgas (Macrocystis pyrifera) y su utilización como ingrediente para la alimentación de salmónidos en la Región de Magallanes y Antártica Chilena" y a la empresa Productos Químicos Algina representada por su gerente regional Sr. Patricio Cáceres. Los autores agradecen a la Dra. Alicia Borazo y al Prof. Héctor Romo por sus valiosas sugerencias y al Dr. Christopher Anderson por la preparación del Abstract.

\section{BIBLIOGRAFIA}

AdAmi, M.L. \& S. GoRdillo 1999. Structure and dynamics of the biota associated with Macrocystis pyrifera (Phaeophyta) from the Beagle Channel, Tierra del Fuego. Scientia Marina 63-1: 183-191.

Alveal, K. 1995. Manejo de algas marinas.En Manual de Métodos Ficológicos (eds. Alveal, K., M. Ferrario, E. Oliveira \& E. Sar), pp. 825-863. Ediciones Universidad de Concepción, Concepción.

Alveal, K., H. Romo \& M. Avila 1982. Estudio del Ciclo de Vida de Macrocystis pyrifera de Isla Navarino, Chile. Revista Gayana Botánica, 39: 1-12.

Buschmann A. H., J. A. Vásquez, E. P. Osorio, E. Reyes, L. Filun, M. C. Hernández-González \& A. Vega 2004. The effect of water movement, temperature and salinity on abundance and reproductive patterns of Macrocystis spp. (Phaeophyta) at different latitudes in Chile. Marine Biology 145: 849-862.

Bushing, W. W. 1997. GIS-based gap analysis of an existing marine reserve network around Santa Catalina Island. International Journal of Marine Geodesy 20: 205-34.

Bustos, E., C. Godoy, S. Olave \& R. Troncoso. 1991. Desarrollo de técnicas de producción de semillas y repoblación de recursos bentónicos. I. Investigaciones en el erizo chileno Loxechinus albus (Molina 1782). Cap. I. PNUD-IFOP (Ed): 1-60.

Candia, A., H. Romo, K. Alveal \& V. Dallarossa 1979. Cultivo unialgal de Macrocystis pyrifera (L) C. 
Agardh de la Bahía de Concepción, Chile. Rickia 8: $75-83$

Cruz-Suárez, L.E., D. Rique-M arie, M. Tapia-Salazar \& C. Guajardo-BA. 2000. Uso de harina de kelp (Macrocystis pyrifera) en alimentos para camarón. Avances en Nutrición Acuícola V. (Eds. CruzSuárez, L. E., D. Ricque-Marie, M. Tapia-Salazar, M.A. Olevera-Nova. \& R. Civera-Cerecedo), pp. 19-22. Memorias del V Simposium Internacional de Nutrición Acuícola, Mérida, Yucatán.

Dayton P. K., M. J.Tegner,P. E. Parnell \&P. B. Edwards 1992. Temporal and spatial patterns of disturbance and recovery in a kelp forest community. Ecological Monographs 62: 421-445.

Drueht, L. 2000. Pacific Seaweeds, A guide to common seaweeds of the west coast. The Canada Council Fort The Arts Since, Canada. 93pp.

GERARD, V.A. 1982. In situ rates of nitrate uptake by giant kelp, Macrocystis pyrifera (L.) C. Agardh: tissue differences, environmental effects, and predictions of nitrogen-limited growth. J Exp. Mar. Biol. Ecol. 62: $211-224$

Graham M. H., C. Harrold, S. Lisin, K. Lght, J. A. Watanabe \& M. S. Foster. 1997. Population dynamics of giant kelpMacrocystis pyrifera along a wave exposure gradient. Marine Ecology Progress Series. 148: 269-279.

Hoffmann, A. J \& B. Santelices. 1997. Flora Marina de Chile Central. Ediciones Pontificia Universidad Católica de Chile, Santiago.434 pp.

Iriarte, J. L., A. Kusch, J. Osses \& M. Ruiz 2001. Phytoplankton biomass in the sub-Antarctic area of the Straits of Magellan (53 $\left.{ }^{\circ} \mathrm{S}\right)$, Chile during spring-summer 1997/1998. Polar Biology 24: 154-162.

LeVring, T. 1960. Contributions to the marine algal flora of Chile. Reports of the Lund University Chile Expedition 1948-49.

Norambuena, R. 1996. Recent trends in seaweed production in Chile. Hydrobiologia 326/327: 371379.

NoRTH, W.J. 1971. Introduction and background. In: W. J. North (ed.). The Biology of Giant Kelp Beds (Macrocystis) in California. Nova Hedwigia. 32: $1-97$.

Mansilla A., M. Palacios, N. Navarro \& V. Riquelme. 2005a. Studies of the populations of Macrocystis pyrifera (L) C. Agardh in Tierra del Fuego, Chile. Report on Polar and Marine Reseach 507: 160162.

M ansilla A., M. Palacios, J.P lana \& N. N Avarro. 2005 b.
Macrocystis pyrifera, una nueva alternativa como aditivo en la alimentación de salmónidos. En Proyecto Fondef Producción de harina de macroalgas (Macrocystis pyrifera) y su utilización como ingrediente para la alimentación de salmónidos en la Región de Magallanes y Antártica Chilena, Punta Arenas, Chile. 7pp.

Ojeda, P.F \& B. Santelices. 1984. Invertebrate communities in holdfast of Macrocystis pyrifera from southern Chile. Marine Ecology Progress Series 16: 65-73.

Palacios, M. \& A. Mansilla. 2003. Desarrollo de gametofitos y esporofitos de Macrocystis pyrifera (L.) C. Agarh (Laminariales: Lessoniaceae) de la Región de Magallanes en condiciones de laboratorio. Anales del Instituto de la Patagonia Ex Serie Ciencias Naturales 31: 43-63.

Pisano, E. 1977. Fitogeografía de fuego-patagonia chilena. I. Comunidad vegetales entre las latitudes 52 y $56^{\circ} \mathrm{S}$. Anales del Instituto de la Patagonia. Ex Serie Ciencias Naturales 8: 121-250.

Ramírez, M. \& B. SAntelices. 1991. Catálogo de las algas marinas bentónicas de la costa temperada del Pacífico de Sudamérica. Monografías Biológicas. $\mathrm{N}^{\circ}$ 5. Facultad de Ciencias Biológicas Pontificia Universidad Católica de Chile.

Romo, H., M. Avila, M. Núñez, R. Pérez, A. Candia, G. Aroca. 2006. Culture of Gigartina skottsbergii (Rhodophyta) in Southern Chile. A Pilot Scale Approach. Journal of Applied Phycology DOI 10.1007/s10811-006-9026-x, URL http:// dx.doi.org/10.1007/s10811-006-9026-x: Pages $1-8$.

VÁsquEZ, J.A. 1999. The effect of harvesting of brown seaweeds: A social, ecological and economical importance resource. Word Aquaculture Magazine 31(1): 19-22.

ZAR, J.H. 1999. Biostatistical analysis. Fourth Edition. Prentice-Hall, Inc. New Jersey: 663 pp.

Westermeier, R., D. Patiño., M.I. Piel, M. Seguel, S. M adariagas, I. Uriarte. 2004. Investigación y desarrollo tecnológico del cultivo de algas y su utilización por invertebrados en Chile. Informe final proyecto Fondef: 152p.

WhEeLER, W. N. 1980. Effect of boundary layer transport on the fixation of carbon by the giant kelp, Macrocystis pyrifera. Mar. Biol. 56: 103-110.

WheELER, W. N. 1982. Nitrogen nutrition of Macrocystis. In L. M. Srivastava (ed.), Synthetic and degradative processes in marine macrophytes. Walter de Gruyter, Berlin: 121-137. 